\title{
Inventaire Floristique des Mauvaises Herbes dans une Caféiculture en Pure dans le Territoire de Kabare, DR Congo
}

\author{
Christus C. MIDERHO ${ }^{1}$, Félicien M. BIRIMWIRAGI ${ }^{1}$, Alain S. KADORHO2 ${ }^{2}$, Benjamin H. BISIMWA3 \\ Dieudonné B. SHAMAMBA ${ }^{3}$, Ignace M. SAFARI ${ }^{4}$, Bossissi G. Nkuba ${ }^{185}$ et Walangululu J. \\ MASAMBA $^{1}$ \\ 1: Département de phytotechnie, Faculté de Sciences Agronomiques, Université Catholique de Bukavu(UCB), B.P. \\ 02 Cyangungu-Rwanda. Phone number: (+243) 977292684 Email: cito.miderho@ucbukavu.ac.cd \\ 2: Laboratoire d'Écologie végétale, Département de Biologie, Faculté des Sciences et Sciences Appliquées, \\ Université Officielle de Bukavu(UOB) \\ 3: Département des Eaux et Forêts, Faculté des Sciences Agronomiques, Université Catholique de Bukavu,(UCB) , \\ B.P. 02 Cyangungu-Rwanda \\ 4: Laboratoire de Earth and Life Institute (ELI), Université Catholique de Louvain (UCL), Louvain-la-Neuve, Belgique \\ 5: Centre d'expertise en Gestion Minière(CEGEMI), Université Catholique de Bukavu(UCB), B.P. 02 Cyangungu- \\ Rwanda
}

Original submitted in on 14th July 2017. Published online at www.m.elewa.org on $30^{\text {th }}$ November 2017 https://dx.doi.org/10.4314/jab.v119i1.7

\section{RÉSUMÉ}

Objectif : Cette étude avait pour objectif d'identifier les mauvaises herbes dans une caféiculture en pure, tout en précisant le degré d'infestation de chacune d'elles afin de planifier une lutte intégrée pour améliorer la production du café.

Méthodologie et résultats :La méthode utilisée dans cette étude est la méthode phytosociologique de Braun Blanquet; où trois plantations ont été enquêtées en réalisant cinq aires minimales de 35 à $90 \mathrm{~m}^{2}$ Ainsi, Une aire minimale de $20 \mathrm{~m}^{2}$ à $80 \mathrm{~m}^{2}$ a été déterminée suivant l'homogénéité de la végétation adventice. C'est ainsi que, 5 aires minimales par plantation ont été enquêtées avec un total de 15 aires minimales et 8 relevés par aire avec un total de 120 relevés enquêtés II a été trouvé que la famille des asteraceae représente $35.29 \%$ et que Bidens pilosa et Galisonga ciliata sont les espèces les plus représentées et présentant un degré d'infestation élevé (adventices majeures dans les caféicultures) par rapport aux autres espèces.

Conclusion et Application des résultats : Cette étude a révélé que la plupart d'espèces caractéristiques des champs de caféier en pure sont des asteraceae (Galinsoga ciliata, Bidens pilosa, Botriocline longipes, etc.) et des poaceae (Digitaria vestida, Setaria barbata, etc.). En plus, Ageratum conyzoidens, Achyranthes asper, Oxalis corymbosa, Crassophalum bumbese, Lactus spp., Cyperus distans, Commelina diffusa, Drymaria cordata, Sida acuta, Galinsoga ciliata et Bidens pilosa sont des espèces nuisibles dans les caféicultures. Ainsi, les résultats de cette étude pourraient être utilisés par les malherbologues afin de mettre en place une lutte focalisée et intégrée contre l'ensemble des mauvaises herbes qui peuvent avoir un impact négatif sur la culture du café et/ou son rendement.

Mots clés : Inventaire, Mauvaises herbes, Caféier, asteraceae, gestion des mauvaises herbes 


\section{ABSTRACT}

Objective: The floristic inventory of weeds in this study aims to identify weeds in a pure coffee plantation, while specifying the degree of infestation of each of them in order to plan an integrated fight to improve the production of coffee.

Methodology and results: The method used for this study is the phytosociological method of Braun Blanquet; where 5 minimum areas of 20 to 80 sqm were analysed in three large coffee farms. In each minimum area 8 quadrats were investigated. The Asteraceae has been found at $35.29 \%$ and Bidens pilosa and Galisonga ciliata are most represented species with a high degree of infestation.

Conclusion and application and results: This study revealed that most characteristic species of pure coffee fields are Asteraceae (Galinsoga ciliata, Bidens pilosa, Botriocline longipes, etc.) and Poaceae (Digitaria vestida, Setaria barbata, etc.). In addition, Ageratum conyzoidens, Achyranthes asper, Oxalis corymbosa, Crassophalum bumbese, Lactus spp. Cyperus distans, Commelina diffusa, Drymaria cordata. Aida acuta. Galinsoga ciliata and Bidens pilosa are harmful species in coffee growing. Thus, the results of this study could be used by weed scientists to put in place a focused and integrated fight against all weeds that can have a negative impact on the coffee culture and / or its yield.

Key words: Inventory, Weeds, Coffee, Asteraceae, weed management

\section{INTRODUCTION}

En République Démocratique du Congo, notamment à l'Est du pays, la culture du café arabica est une source importante de revenus pour un grand nombre de producteurs agricoles. Dans cette région, le caféier arabica est prédominant malgré que la surface emblavée reste moyennement faible, avec une superficie de moins de un hectare par producteur (Lacs, 2014). La culture du café est soumise à des nombreuses contraintes biotiques (parasitaires) et abiotiques contribuant à la baisse des rendements. En effet, les pertes de la productivité des systèmes pérennes à base du café au Sud Kivu sont dues au manque d'intrants agricoles; le manque d'encadrement technique des petits producteurs; aux maladies telles que la rouille, l'anthracnose; aux ravageurs tels que le puceron noir du caféier ; ainsi qu'aux adventices (Zaire n.d.). Les mauvaises herbes constituent une des contraintes majeures dans la culture du caféier. Elles causent une perte de rendement pouvant dépasser $25 \%$ en zone tropicale (Boudjedjou, 2010). Ce sont des plantes prolifiques et envahissantes de part leur capacité à produire une grande quantité des graines. Ces plantes entrent en compétition pour l'eau et en nutriment avec la culture principale; et constituent un réservoir pour un certain nombre de virus communs. En plus, les adventices abritent de nombreux nématodes et insectes ravageurs tels la punaise, les cicadelles, et les pucerons sont particulièrement attirés par elles. Enfin, elles sont parfois des plantes allergènes ou allergisantes (Anonyme, 2010). Lacey (1985) et Vincent et Panneton (2000) avaient prouvé que la compétition entre un certain nombre des plantes et les mauvaises herbes entraînent des pertes importantes de rendement allant de $24 \%$ à $99 \%$. D'une manière générale, les pertes avant récolte sont de l'ordre de 20 à $40 \%$ tandis que les pertes post-récolte (denrées stockées) représentent 10 à $20 \%$ (Riba et Silvy, 1989, Boudjedjou, 2010). Selon FAO (2009), la perte de production suite aux mauvaises herbes est évaluée à plus de 95 millions de dollars américains par an dans lequel plus $70 \%$ proviennent dans les pays pauvres (Quee, 2016). Cependant, la culture du caféier n'est pas épargnée surtout en ce qui concerne sa qualité lors de sa transformation. Les mauvaises herbes appartiennent à plusieurs familles et possèdent donc de variabilité biologiques et génétiques salon l'espèce et les écosystèmes où elles se trouvent (Sciences et al., 2012). Kazi et al (2010) avaient montré que la connaissance de la composition de la flore des mauvaises herbes et son évolution sous l'effet de des facteurs environnementaux et agronomiques serait un préalable indispensable à toute mise au point de stratégies de lutte intégrée afin d'obtenir des plants 
au jeunes âge, une fructification maximale, un ramassage et une récolte facile des fruits et ainsi qu'une bonne résolution du problème de la perte de la qualité et la quantité des produits de culture (Tani et al., 2011), (Traore et al.,2005). Cependant, Les mauvaises herbes appartiennent à plusieurs familles et possèdent de biologies très variées d'une espèce à une autre en raison de leur écologie et physiologie (Sciences et al., 2012). En 2010 , Kazi , Le Bourgeois et Munoz montrent que la connaissance de la composition de la flore des mauvaises herbes et son évolution sous l'effet des facteurs environnementaux et agronomiques est un préalable indispensable à toute mise au point de stratégies de lutte intégrée afin d'obtenir des plants au jeunes âge, une fructification maximale et de faciliter la récolte et le ramassage des fruits et ainsi résoudre le problème de la perte de qualité et quantité des produits de culture.(Tani et al.,2011), (Traore et al., 2005). L'élimination de la végétation adventice dans une

\section{MATÉRIELS ET MÉTHODES}

Milieu d'étude: La présente étude a été réalisée à Bugorhe, un groupement situé dans la partie Nord du territoire de Kabare. II s'étend entre $28^{\circ} 45^{\prime}$ à $28^{\circ} 85^{\prime}$ de longitude est et de $2^{\circ} 15^{\prime}$ à $2^{\circ} 30^{\prime}$ de latitude sud, et situé entre $1470 \mathrm{~m}$ et $2200 \mathrm{~m}$ d'altitude (Figure 1) (Balagizi et al., 2013). L'inventaire a été réalisé dans trois plantations de café en monoculture, situées dans le groupement de Bugorhe et Bushumba dont la plantation Bwengehera (70 hectares), la plantation Kavea (74 hectares) et la plantation Justin Ntaboba (53 hectares).

Méthodes: La Méthode utilisée pour faire cet inventaire est celle de Braun-Blanquet (Meddour, 2011). Les aires minimales ont été déterminées selon l'homogénéité de la végétation (Gillet, 2000, Dellassus, 2015) et choisies aléatoirement, leur nombre par plantation était de cinq. Chacune d'elle mesurant $35 \mathrm{~m}^{2}$ et $90 \mathrm{~m}^{2}$ (Delpech, 2006); et 8 quadras de $1 \mathrm{~m}^{2}$ étaient plantation a pour effet d'obtenir un meilleur développement des plants au jeune âge, puis une fructification maximale et de faciliter la récolte et le ramassage des fruits détachés. En plus, avec la rareté de la main d'œuvre et le cout élevé des traitements herbicides. Face à tous ces problèmes que posent les adventices, il est important qu'une stratégie de lutte intégrée de l'enherbement soit mise en œuvre d'où l'importance d'une meilleure connaissance de la diversité floristique et de sa dynamique (Traore et al. , 2005, Assani et al., 2015). Malheureusement, au Sud-Kivu, malgré l'importance que présente la culture du caféier, peu de données, voir même pas, sur les adventices du caféiculture sont disponible. L'objectif de ce travail est d'inventorier les Mauvaises herbes trouvées dans une caféiculture en pure afin d'identifier ces adventices pour ainsi contribuer à l'augmentation de la valeur qualitative et quantitative du Café du Kivu.

étudies par aire minimale. Ainsi, une somme totale de 120 relevés a été atteinte. La procédure a consisté à prélever des échantillons suivant l'approche traditionnelle de Braun Blanquet avec une échelle d'abondance dominance de 1 à 100\% correspondant au recouvrement du sol. Selon Lahondere (1999) et Gillet(2000), l'indice d'abondance-dominance est une estimation globale de la densité (nombre d'individus, ou abondance) et du taux de recouvrement (vu de dessus de la partie aérienne des végétaux, ou dominance) des éléments de la synusie (organismes individuels représentant l'idiotaxon élémentaire) dans l'aireéchantillon. Les taux de recouvrement sont estimés en proportion de la surface effectivement recouverte par l'ensemble des végétaux de la synusie relevée (Sv), et non en proportion de la surface totale du relevé (St), contrairement à l'usage classique inauguré par BraunBlanquet (1928). 


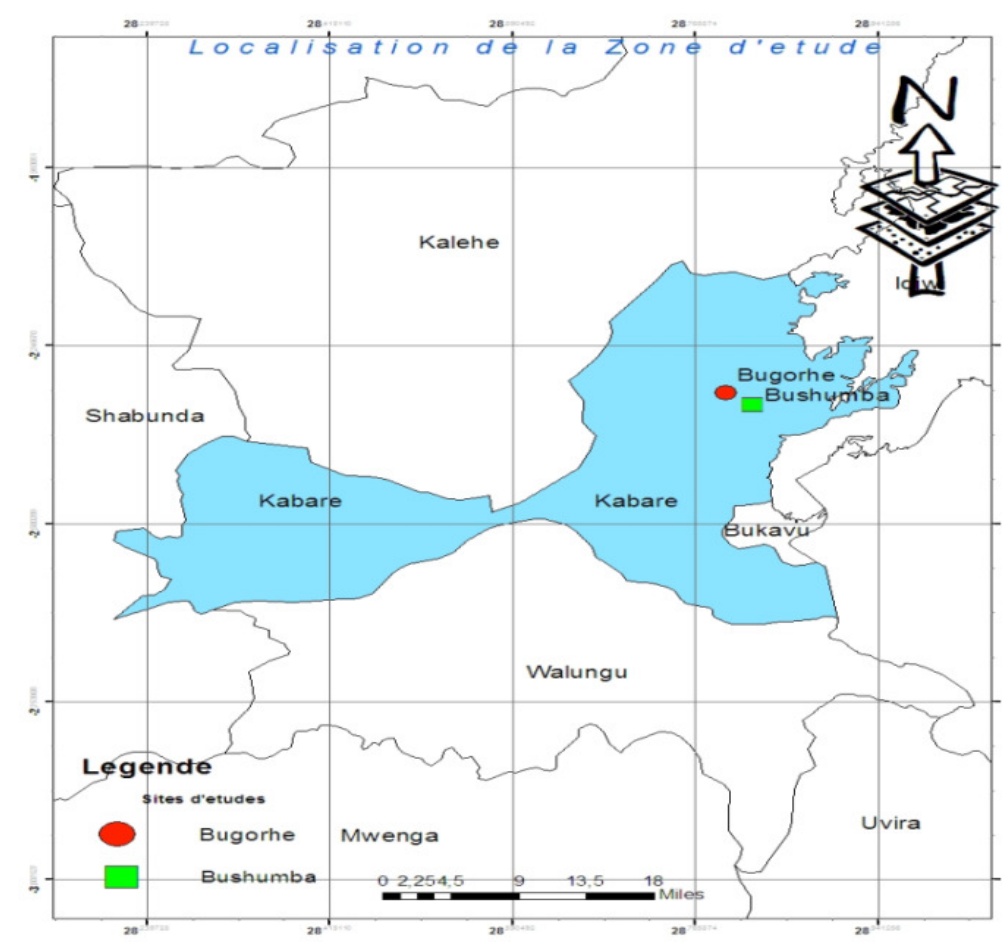

Figure 1. Localisation du milieu d'étude

On distingue les classes suivantes:

$r$ :éléments uniques ou très peu abondants, recouvrement inférieur à $1 \%$ de $S v$

+:Éléments peu abondants, recouvrement inférieur à $5 \%$ de $S v$

1 : élément assez abondant, recouvrement inférieur à $5 \%$ de $S v$

2 : éléments très abondants, recouvrement inférieur à $25 \%$ de Sv

3: recouvrements compris entre 25 et $50 \%$ de Sv, abondance quelconque

4: recouvrements compris entre 50 et $75 \%$ de Sv, abondance quelconque

5 : recouvrements supérieurs à $75 \%$ de $S v$, abondance quelconque.

Certains auteurs tel que Barkman et al.(1964) ; Westhoff et Van der maarel (1978) et Theurillat (1992) ont proposé trois subdivisions pour l'indice 2:

$2 m$ : éléments très abondants, recouvrement inférieur à $5 \%$

$2 a$ : recouvrement compris entre 5 et $12.5 \%$, abondance quelconque

$2 b$ : recouvrement compris entre 12.5 et $25 \%$, abondance quelconque

L'indice $2 m$ est difficile à distinguer de l'indice 1 . II nous paraît plus judicieux de restreindre l'usage de l'indice 2 à des espèces dont le recouvrement dépasse $5 \%$ (2a et $2 b) ; 1$ et $2 m$ seront donc regroupés sous l'indice 1 (Gillet, 2000). L'inventaire Floristique a été fait sur les parties aériennes et cela par la mise en place d'un quadrat de $1 \mathrm{~m}^{2}$ qui est subdivisé en 100 petits carrés posés aléatoirement dans le champ suivi immédiatement d'un inventaire floristique au sein de chaque cadre et une étude phytosociologique. Cependant, les espèces non identifiées directement sur terrain ont été prélevés afin qu'elles soient identifiées à l'herbarium du Centre de Recherche en Sciences Naturelles de Lwiro (C.R.S.N/LWIRO).

Fréquence relative : La fréquence relative $(\mathrm{Fr})$ d'une espèce végétale donnée se définit comme le rapport de sa fréquence absolue $(\mathrm{Fa})$ ou nombre de relevés où elle est présente au nombre total ( $\mathrm{N}$ ) de relevés effectués sur un site donné. Elle se traduit par l'expression suivante :

$\operatorname{Fr}(\%)=(\mathrm{Fa} / \mathrm{N}) .100$

La répartition des espèces selon leurs fréquences relatives dans chaque site a été déterminée conformément à l'histogramme de Raunkiaer (1905) cité par Mangara et al. (2008). Les classes de fréquences relatives des taxons selon Raunkiaer (1905) sont présentées dans le tableau. 
Tableau 1 : Classes de fréquences relatives selon Raunkiaer

\begin{tabular}{l|l}
\hline Classe de fréquence & fréquences relatives \\
\hline Classe I & 0 à 20 \\
\hline Classe II & 21 à 40 \\
\hline Classe III & 41 à 60 \\
\hline Classe IV & 61 à 80 \\
\hline Classe V & 81 à 100 \\
\hline
\end{tabular}

Coefficient de similitude (CS) : Ce coefficient permet de vérifier l'homogénéité des sites de relevés pris deux à deux au regard de leur composition floristique. II est déterminé selon la formule de Sorensen (1948) ci-après

\section{$C s=100.2 c /(a+b)$}

Où $a$ et $b$ représentent les nombres d'espèces recensées respectivement dans les deux sites échantillonnés, $c$ étant le nombre d'espèces communes aux deux sites $A$ et $B$. Le coefficient de similitude (Cs) varie de 0 à $100 \%$ selon que les deux sites sont de compositions floristiques totalement différentes $(c=0)$ ou identiques $(a=b=c)$. Pour un coefficient de similitude supérieur ou égal à $50 \%$, les deux sites concernés sont considérés comme floristiquement identiques.

Diagramme d'infestation: Ce diagramme permet de classifier les mauvaises herbes suivant leur pression sur la culture principale. Cette pression est déterminée par deux méthodes: L'une tient à compte la contribution spécifique et la contribution floristique et l'autre basé sur la fréquence relative et le coefficient d'abondance dominance. Pour ce présent travail, la seconde méthode sera utilisée. Tout commence par la catégorisation des mauvaises herbes et leur traduction en degré d'infestation c'est-à-dire leurs fréquences et leurs indices d'abondance-dominance moyenne sur l'ensemble des sites étudiés. Elles sont fonction de la fréquence absolue et du recouvrement global (Marnotte, 1989 ; Traoré, 1991, Tourré et al ,2008) et qui sont présentées en 7 groupes d'adventices (frm: fréquence relative moyenne ; IADm: Indice d'Abondance-Dominance moyenne) :

- Les adventices majeures de la zone: frm> $50 \%$, IADm entre ]2, 4] sur la zone ;

- Les adventices majeures locales : frm> $50 \%$, IADm entre ]2,4] sur le site ;

- les adventices majeures potentielles de la zone :Frm appartient dans l'intervalle $[24,50] \%$;

ADm compris]1,2]; sur la zone ;
- $\quad$ Les adventices majeurs potentiels locaux :Frm appartient dans l'intervalle $[24,50]$ et IADm appartient dans ]1 , 3 ] sur le site ;

- Les adventices accompagnatrices de la zone Frm appartient dans l'intervalle $[10,24] \%$;; et IADm appartient dans l'intervalle]-infinie, 1] sur la zone ;

- Les adventices accompagnatrices du site: Frm entre $[10,24] \%$; ; et IADm appartient dans]-infinie, 2] sur le site ;

- Les adventices mineures ou accidentelles 10 $<$ Frm, $+{ }^{\wedge} \mid \mathrm{ADm}{ }^{\wedge} 4$ sur le site ;

Quantification de la biodiversité sur les sites en étude : Indices de diversité floristique

Afin d'évaluer la richesse spécifique de nos sites, l'indice de diversité de Shannon-Weaver et l'indice de diversité de Simpson ont été utilisés. Ces indices s'expriment de la manière suivante (Grall et Hily, 2003) Indice de Shannon-Weaver $\left(\mathrm{H}^{\prime}\right)$ :

$H^{\prime}=-\sum_{i=1}^{g}\left(\frac{n i}{N}\right) \log 2\left(\frac{n i}{N}\right)$

Où:ni -le nombre d'individus d'une espèce dans l'échantillon,

$N$-le nombre total d'individus de toutes les espèces dans l'échantillon,

$S$-le nombre total d'espèces.

H' s'exprime en bits et varie de 0 à log2S, soit environ 4,5 pour une communauté assez riche (Bouko et al., 2007),.

L'indice de Shannon-Weaver est minimal $\left(H^{\prime}=0\right)$ si tous les individus du peuplement appartiennent à une seule et même espèce, $H^{\prime}$ est également minimal si, dans un peuplement chaque espèce est représentée par un seul individu, excepté une espèce qui est représentée par tous les individus du peuplement. L'Indice de ShannonWeaver $\left(\mathrm{H}^{\prime}\right)$ est maximal quand tous les individus sont répartis d'une façon égale sur toutes les espèces (Issoufa et al., 2008, Pedel et Fabri, 2012). 
L'indice d'équitable de Piélou(J'): cet indice accompagne souvent lindice de Shannon. II permet de mesurer la répartition des individus au sein des espèces. Compris entre 0 et 1 , les individus sont équirépartis dans les espèces lorsque la valeur de J'est égale à 1 .

\section{$\mathrm{J}^{\prime}=\boldsymbol{H}^{\prime} / \mathrm{H} \max$}

Avec Hmax $=\log _{2} S$ et $S=$ le nombre d'espèces. Cet indice permet d'apprécier le désordre relatif de la population de Mauvaises herbes.

Types biologiques: Les types biologiques ont été identifiés sur le terrain par l'observation respective des ports végétatifs et position des bourgeons de régénération des espèces de la flore dénombrée. Pour ce faire, la classification de Raunkier (1934), adoptée aux régions tropicales par Lebrun (1966), a été utilisée. Suivant cette classification, nous avons retenu les types biologiques (Reprenant, 1904) (Smith, 2013) suivants : Les Phanérophytes $(\mathrm{Ph})$ : plantes dont l'appareil caulinaire porte à plus de $40 \mathrm{~m}$ du sol les bourgeons ; Ainsi, nous avions trouvé Le Nanophanérophytev (Naph)

\section{RE SULTATS ET DISCUSSION}

Liste Floristique des adventices inventoriés dans la caféiculture: Au cours de cette étude, nous avons recensé 18 espèces (tableau 2) considérées comme adventices des caféicultures. Ces espèces sont
Les Chaméphytes (Ch): plantes ayant l'appareil végétatif d'une hauteur inférieure à $40 \mathrm{~cm}$, avec les bourgeons protégés par le débris végétaux. Nous avions trouvé: Chaméphyte herbacée (Cherb), Chaméphyte rampant (Chra) et Chaméphyte grimpant (Chgr)

Les Hémicryptophytes $(\mathrm{Hc})$ : plantes dont les bourgeons persistant sont enfouis dans le sol.

Les Thérophytes $(T)$ : Plantes qui persistent sous formes de graines. Nous avions trouvé: Le Thérophytescapeux(Tsc) : sont des plantes subsistant qu'à l'état de graine et ayant un port érigé. ou dressé, Thérophytes cespiteux (Tces): plantes vivant en touffe.

Les Géophytes rhizomenteux (Grh): Plantes dont la partie pérenne de l'appareil végétatif est profondément enfouie dans le sol, les bourgeons hivernants sont sur un rhizome ou tige souterraine, à croissance mono ou sympodiale.

Analyses statistiques de données : Les données ont été soumises à l'analyse en composante Principale afin de classifier les mauvaises herbes selon leur nuisibilité dans les caféicultures. A cet effet, les logiciels R 2.10.1 avec l'utilisation du package FacterMineR et Past ont été utilisés pour analyser les données.

groupées dans leurs familles, dans leurs genres respectifs en ordre alphabétique. Chaque espèce est affectée de son type biologique.

Tableau 2 : Liste floristique des adventices inventoriée

\begin{tabular}{l|l}
\hline T.B : Type Biologique & Types biologiques \\
\hline Familles et Espèces & \\
\hline Amarantaceae & T. Sc \\
\hline 1. Achyranthes asper L.T. & \\
\hline Asteraceae & T. Sc \\
\hline 2. Ageratum conyzoides L.T. & T. Sc \\
\hline 3. Bidens pilosa L.T. & Chher \\
\hline 4. Crassocephalum bumbes & T. Sc \\
\hline 5. Galinsoga ciliata(RAF.) BLAKET. & T. Sc \\
\hline 6. Bothrioclinelongipes T. & T. Sc \\
\hline 7. Lactuca inermisT. & \\
\hline Cannaceae & Grh \\
\hline 8. Canna indicaL. & \\
\hline Caryophylaceae & Chher \\
\hline 9. Drymariacordata(L.) WILD. EX ROEM ET SCHULT & \\
\hline Commelinaceae &
\end{tabular}


Miderho et al., J. Appl. Biosci. 2017 Inventaire Floristique des Mauvaises Herbes dans une Caféiculture en Pure dans le Territoire de Kabare, DR Congo

\begin{tabular}{l|l}
\hline 10. Commelina diffusa Burm.F. & Chsuc \\
\hline Cyperaceae & \\
\hline 11. Cyperus distensL.f. & Grh \\
\hline Malvaceae & \\
\hline 12. Sida acuta Brum.F. & Chsuf \\
\hline Nyctaginaceae & \\
\hline 13. Mirabilis jalapa L. & Grh \\
\hline Oxalidaceae & \\
\hline 14. Oxalis corymbosa DC.T. & T. Sc \\
\hline Poaceae & \\
\hline 15. Digitaria vestida DENOT var. Sclarum (HOCHST. ex A. RICH.STAPF) & Grh \\
\hline 16. Imperata cylindrica(L.) P Beauv. & T. Ces \\
\hline 17. Setaria barbata (CHAM.) KUNTH.T.ces & T. Sc \\
\hline Polygonaceae & \\
\hline 18. Rumex abyssinica & Chher \\
\hline
\end{tabular}

Familles les mieux représentées dans les caféicultures: le cortège floristique est en majorité constitué des Angiospermes. Les 18 espèces sont réparties dans 18 genres, 11 familles, 7 ordres, 4 sousclasses, 3 classes, 2 sous-embranchements et 1 embranchement. Certaines familles ont beaucoup plus d'espèces et des genres que d'autres. C'est le cas de la famille Asteraceae avec 6 espèces (soit $35,29 \%$ ) réparties en 6 genres (soit $35,29 \%$ ), la famille Poaceae avec 3 espèces (soit 17,65 \%) reparties en 3 genres (soit 17, $65 \%$ ). Les autres familles sont représentées chacune avec 1 espèce (soit 5,88 \%).

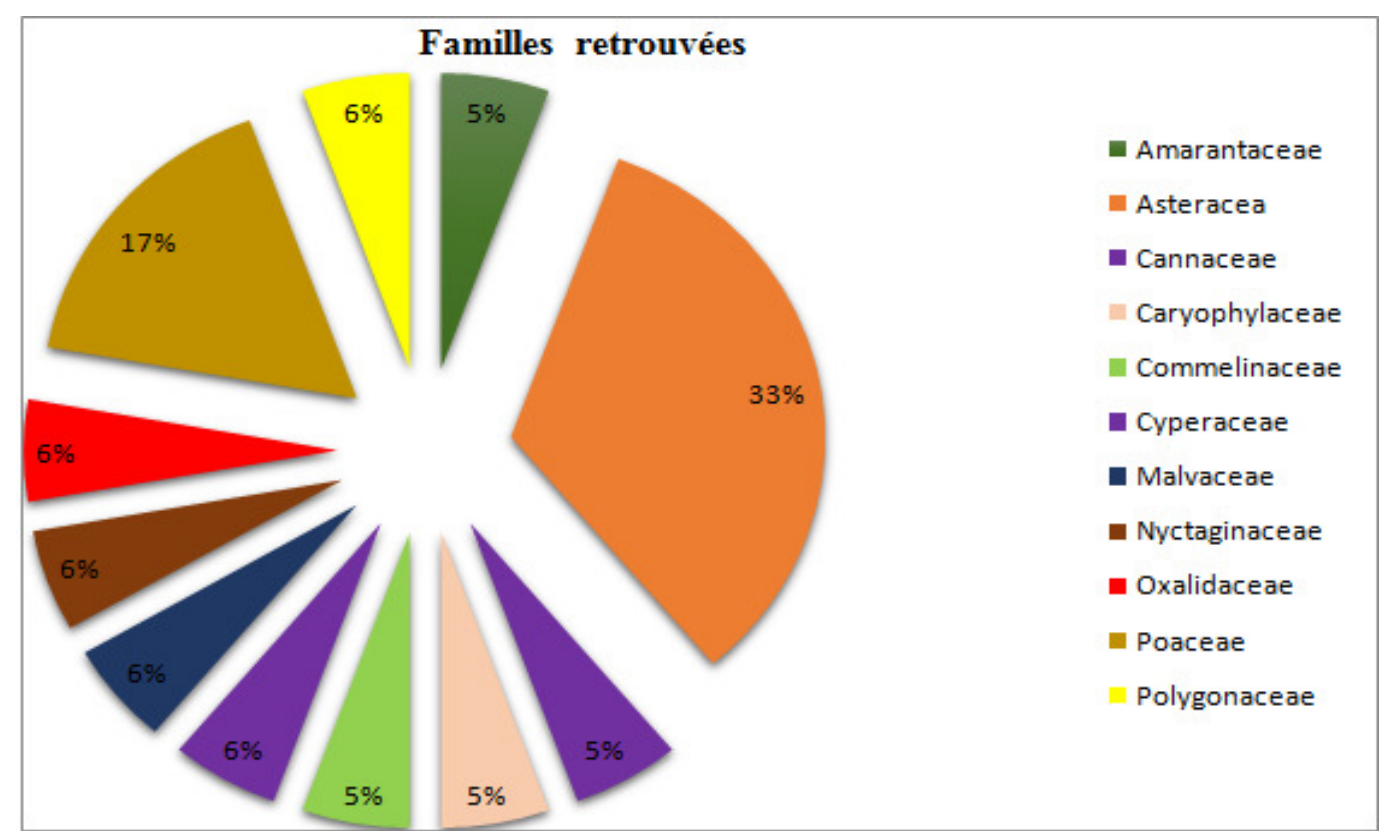

Fig. 1 : Les familles représentées dans les trois plantations en étude

Type biologique : Les Thérophytes dominent la flore étudiée $(44,44 \%)$ suivis respectivement des Cham ephytes $(33,33 \%)$ et Géophytes $(22,22 \%)$. Les caféicultures étudiées sont dominées par des espèces qui passent la mauvaise saison sous forme des graines. 


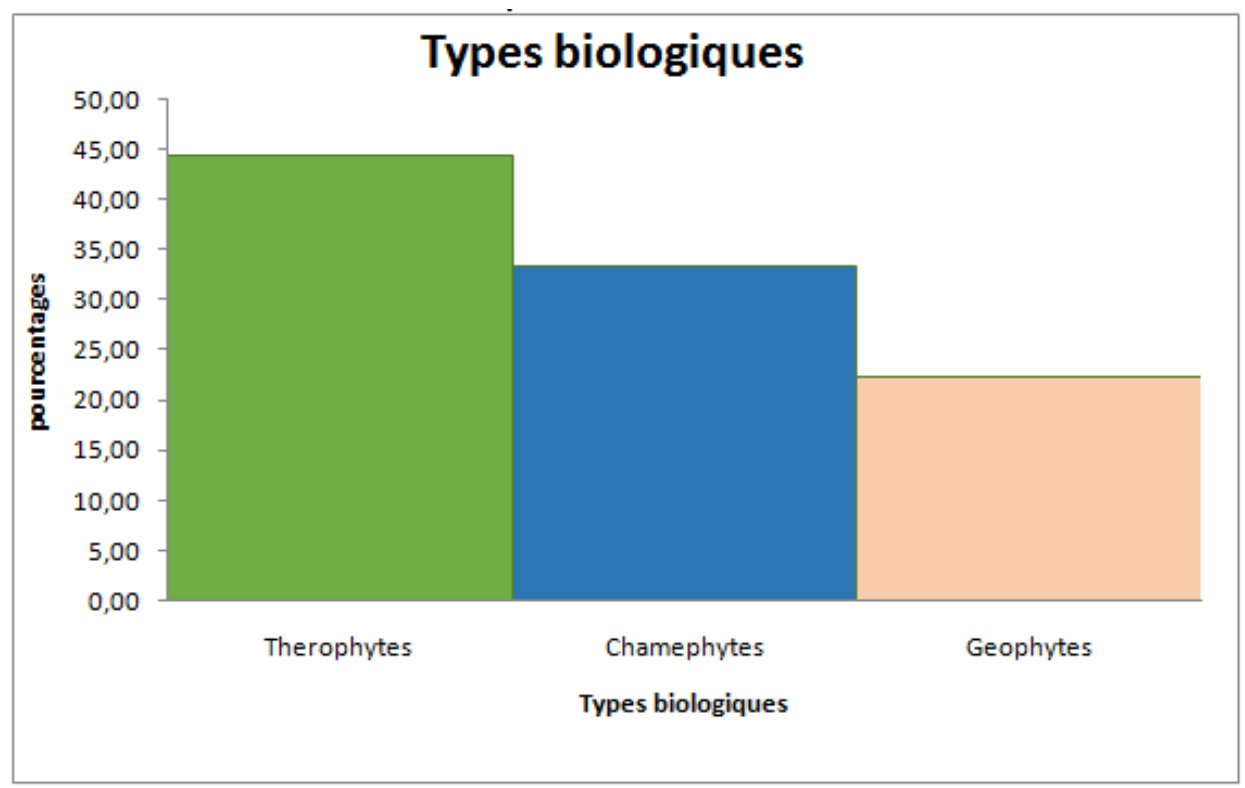

Fig. 2 : Types biologiques

Classes de fréquences relatives : Les classes de fréquences relatives en fonction de plantations sont présentées dans le tableau 3

Tableau 3: Classes de fréquences relatives

\begin{tabular}{|c|c|c|c|}
\hline \multirow[t]{2}{*}{ Classe } & \multicolumn{3}{|l|}{ Plantations } \\
\hline & Bwegerera & Kavea & Justin Ntaboba \\
\hline I & $\begin{array}{l}\text { Ageratum conyzoides, Canna } \\
\text { Drymaria cordata, Imperata } \\
\text { cylindrica, Setaria barbata, Sida } \\
\text { acuta, Rumex abyssinica, Oxalis } \\
\text { corymbosa }\end{array}$ & $\begin{array}{l}\text { Myrabilis jalapa, Ageratum } \\
\text { conyzoides, Commelina } \\
\text { diffusa,Cyperus distens, } \\
\text { Drymaria cordata, Imperata } \\
\text { cylindrica, Lactucainermis, } \\
\text { Myrabilis jalapa }\end{array}$ & $\begin{array}{l}\text { Commelina diffusa, } \\
\text { Crassocephalum bumbese, } \\
\text { Cyperus distens,Imperata } \\
\text { cylindrica,Myrabilis jalapa, Setaria } \\
\text { barbata }\end{array}$ \\
\hline II & $\begin{array}{l}\text { Commelina diffusa, Cyperus } \\
\text { distens, Digitaria } \\
\text { vestida, Galinsoga ciliata, , } \\
\text { Myrabilis jalapa }\end{array}$ & $\begin{array}{l}\text { Bidenspilosa, Crassocephalum } \\
\text { bumbese }\end{array}$ & $\begin{array}{l}\text { Achyranthes aspera, Ageratum } \\
\text { conyzoides, Galinsoga cilliata, } \\
\text { Bothriocline longipes }\end{array}$ \\
\hline III & - & $\begin{array}{l}\text { Digitaria vestida, Galisonga } \\
\text { cilliata, Bothriocline longipes }\end{array}$ & Bidens pilosa \\
\hline IV & - & - & - \\
\hline V & Bidens pilosa & - & - \\
\hline
\end{tabular}

La distribution des espèces selon l'histogramme de Raunkiaer(Mangara et al., 2008) : L'histogramme (fig. 4) se caractérise par une fréquence faible (Classe I) avec un très grand nombre d'individus dans les trois plantations d'une part, et d'autres parts par une forte fréquence (Classe $V$ ) avec un petit nombre d'individus (1 individu dans la plantation de Bwegerera). Les mauvaises herbes moins nuisibles sont représentées dans les classes I avec le nombre maximum des individus 8, 9 et 7 respectivement dans les plantations de Bwegerera, Kavea et Justin Ntaboba tandis que les mauvaises herbes qui pourraient être nuisibles à la culture principale auxquelles les mesures de lutte devraient être prises se retrouvent dans les classes II,III, et V. 


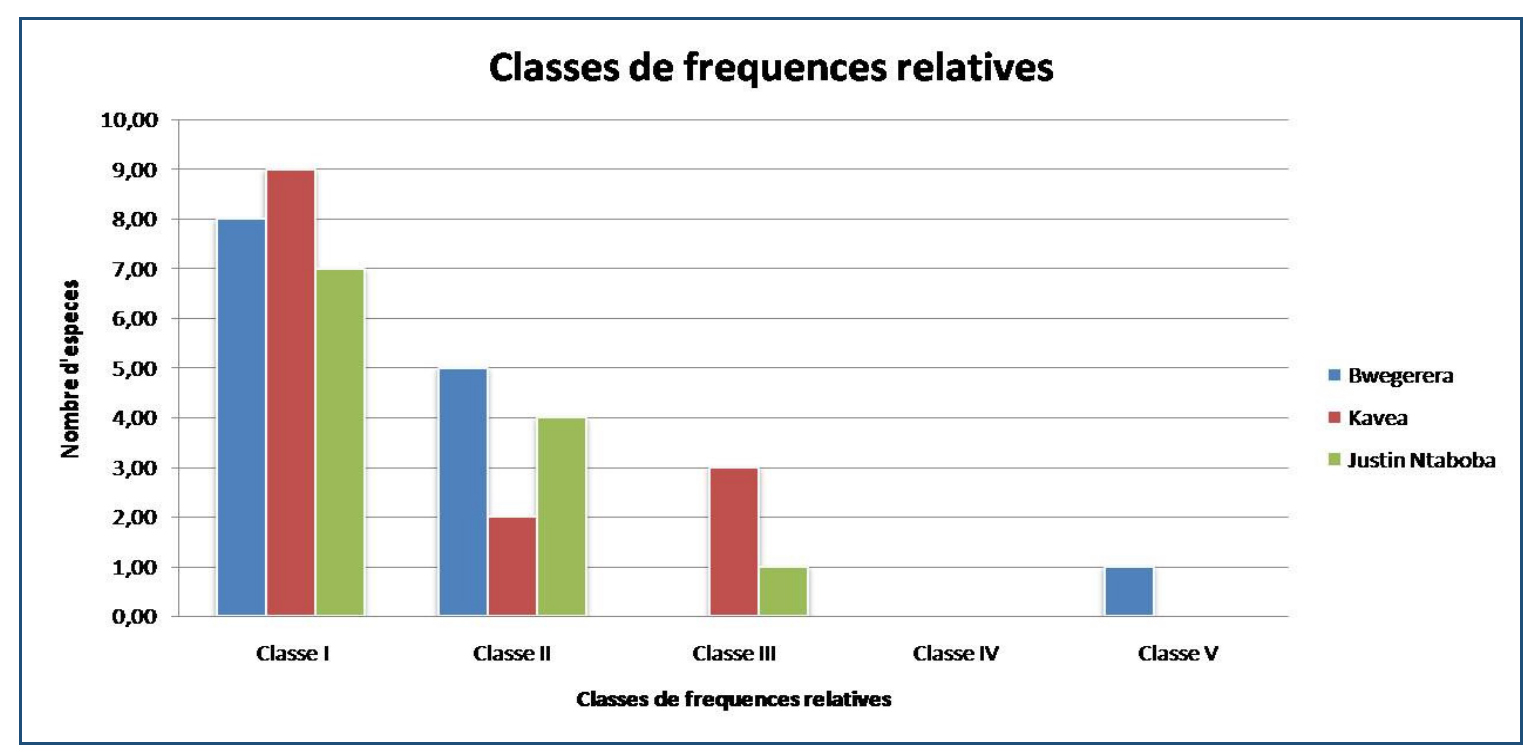

Fig. 4 : Classe de fréquences relatives

Indices de similitude : Le nombre d'espèce dans les plantations Bwegerera et Justin Ntaboba est, respectivement, de 14 espèces contre 13 dans la plantation Kavea. Les nombres d'espèces communes aux plantations sont respectivement de 9 entre Kavea et Bwegerera, 10 entre Kavea et justin Ntaboba et, enfin, 9 entre Justin Ntaboba et Bwegerera. L'indice de similitude est de 0,64 entre les plantations Kavea et Bwegerera et 0,74 respectivement entre les plantations Bwegerera - Justin Ntaboba et Kavea - Justin Ntaboba. Sur base de ces valeurs, ces trois sites sont homogènes du point de vue de la diversité spécifique des adventices qui se développe sous le caféier.

Tableau 4: Indice de similitude entre les différentes stations du site

\begin{tabular}{|l|l|}
\hline Plantation & Indices de similitudes \\
\hline Bwegerera-Kavea & 0,642 \\
\hline Bwegerera-Justin Ntaboba & 0,741 \\
\hline Kavea-Justin Ntaboba & 0,741 \\
\hline
\end{tabular}

Diagramme d'infestation: Le degré d'infestation est apprécié par le diagramme d'ACP (fig. 5), formé à partir de données de fréquence relatives et les indices abondance-dominances, montre trois groupes d'espèces reflétant le degré de nuisibilité de l'espèce tel que le montre la figure 5 .Ainsi : Le Groupe 1 est formé des adventices majeures du site dont Bindens pilosa et Galinsoga ciliata suivi du groupe 2 formé a sont tour par des adventices accidentelles dont Crassophalum bumbese, Achyranthes asper, Ageratum conyzoides, Cyperus Distens et Commelina diffusa et enfin le Groupe 3 est formé des adventices accompagnatrices dont Sida acuta, Digitaria vestida, Imperata cylindrica, Lactuca inermis, Bothriocline longipes, Oxalis corymbosa, Rumex abyssinica, Setaria barabata, Drymaria cordata et Mirabilis jalapa. 


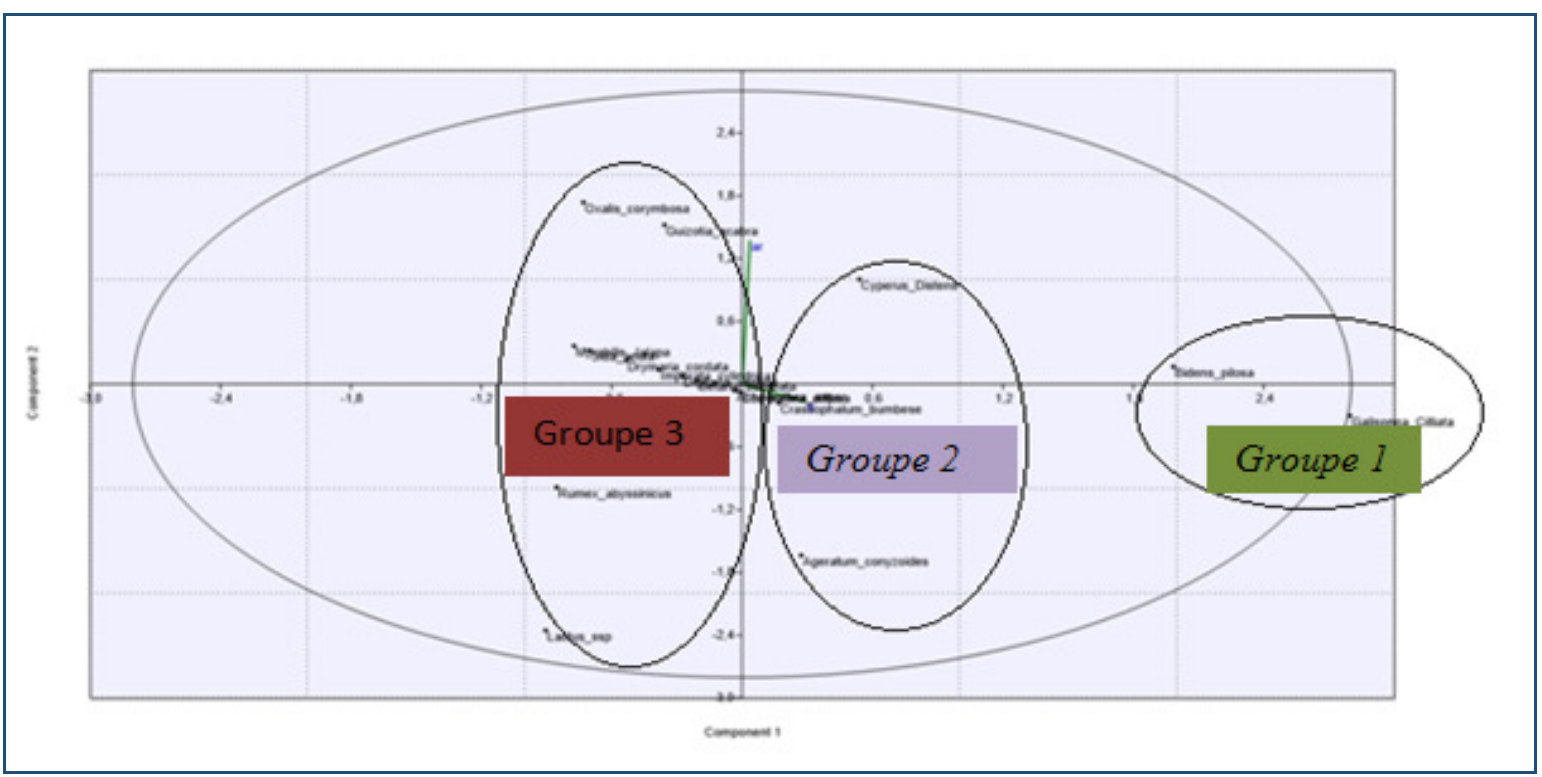

Fig. 5 : Diagramme d'infestation

La valeur propre de la première composante donne la majorité de l'information, qui est de $51.14 \%$ et la Seconde donne moins d'information $(0.67 \%)$, Le pourcentage de la variance est de $88,35 \%$ pour la première composante et de $11,655 \%$ pour la deuxième composante.

Indice de Shannon-Weaver et l'indice d'Equitabilité de Pielou: Oxalis corymbosa a pour indice de Shannon-Weaver une valeur proche de 3.5 ( valeur maximale parmi les espèces inventoriées) (figure 6 ) qui montre qu'il a plusieurs especes dans ces agro écosystèmes cafeicole par rapport à celle d'achyranthes asper est qui est de 1 (valeur minimale parmi les espèces trouvées) et pour l'indice d'équitabilité piélou la plus grande valeur est celle de Galisonga ciliata est approximative à 1 et la plus petite est approximativement égale à 0 pour setaria barbata cela veut dire que pour Galinsonga Ciliata, les individus sont equi-repartie pendant que pour la valeur zéro ces individus sont mal repartie au sein de l'espèce

Indice de Shannon-Weaver et indice d’équitabilité de Piélou

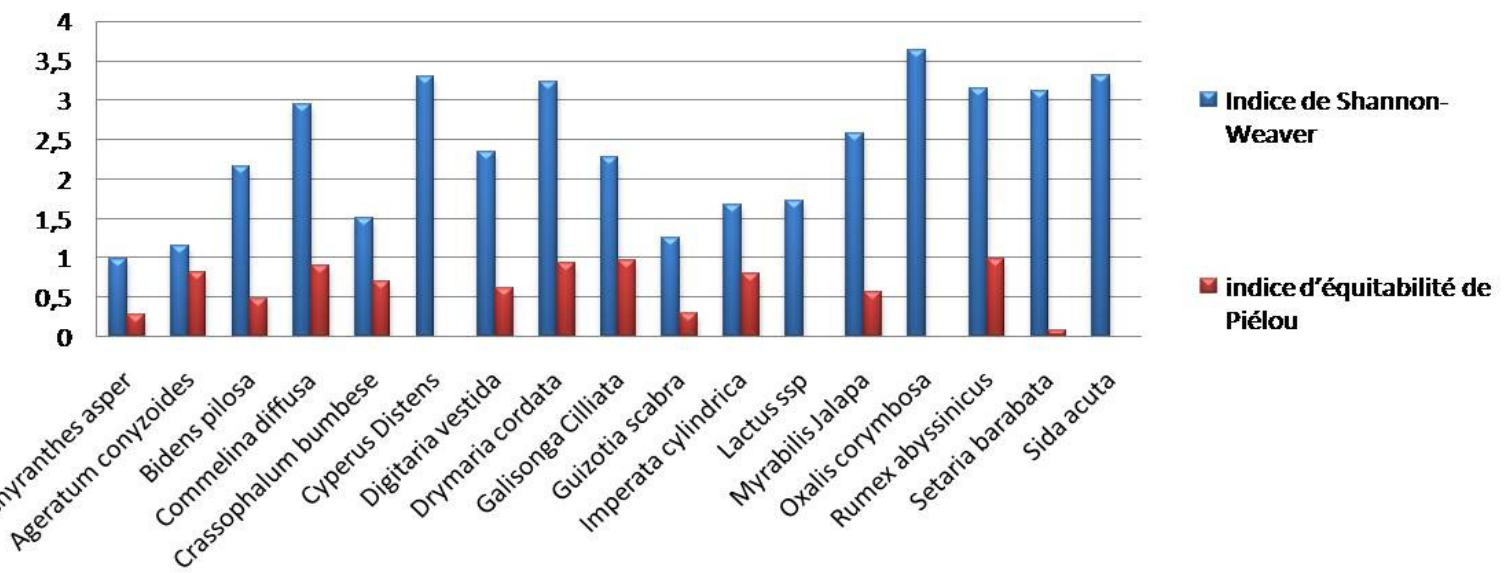

Fig. 7 : Indice de Shannon-Weaver et indice d'Equitabilité de Piélou 


\section{DISCUSSION}

Les relevés réalisés dans les trois plantations de café en pure du groupement de Bughore, ont abouti à l'identification de 11 familles botaniques. Certaines familles se retrouvent dans les 10 familles mauvaises herbes les plus retrouvées dans un agro écosystème à travers le monde. II s'agit des Euphorbiaceae, Malvaceae, Asteraceae, Poaceae, Cyperaceae, Convolvulaceae, Fabaceae, Polygonaceae, Amaranthaceae et Solanaceae (Kouakou et al. 2016). Les familles retrouvées comptent 18 espèces de mauvaises herbes, dont Bindens pilosa et Galinsoga ciliata comme des espèces prépondérantes appartenant à la famille des Astéracées. Ces résultats sont semblable à ceux trouvés par (Flore et al. 2004, Tani et al. 2011, Tehri et Garhwal 2015, Malakand 2015, Quee et al. 2016). Ceci est dû par le fait que $80 \%$ des mauvaises herbes se reproduisent par graines, se disséminent par plusieurs voies comme le vent, l'eau et surtout les insectes, qui fait à ce qu'elles dominent plusieurs écotypes. Comme exemple des Asteracea, qui se caractérisent par la production des petites graines légères souvent et avec une très forte facilité à ses disséminer même par le vent. C'est le cas de Bidens Pilosa, une asteracea qui produit entre 3000 6000 graines ((Bourgeois et Marnotte, 2002). En plus, Osawaru et al, 2014, expliquent que l'abondance de mauvaises herbes peut être le résultat de l'activité anthropique en relation directe ou indirecte avec l'environnement. Selon Brun 2007, affirme aussi qu'une partie des espèces annuelles sont des bisannuelles facultatives cela veut dire que selon les caractéristiques de leur milieu de vie, elles peuvent réaliser leur cycle de vie dans une ou deux ans, qui une graine qui pouvait germer au $t=t_{0}$ germe alors au temps $t=t_{0}+1$ dans le milieu cultivé. Cela peut faire à ce que certaines graines colonisent une zone donnée. Selon plusieurs études qui affirment que selon dans un milieu cultivé, 225 espèces de mauvaises herbes sont souvent présentes et les familles les plus présentées sont Asteraceae, Brassicaceae, Poacea, caryophylacea et Fabacea (Bourgeois et Marnotte, 2002). En plus, La présence des autres espèces est plus due à leur capacité d'adaptation. Dans une culture l'ordre suivant est respecté: Th $>$ Cha $>$ Geo. Les Therophytes sont plus présentes dans ces plantations que les Chamephytes et ces dernières sont plus que les Geophytes. Ces résultats sont semblable à ceux de (Onana et al., 2007) (Tani et al. 2011), qui, dans une population de mauvaises herbes dans une culture, ont trouvé que les Therophytes étaient majoritaires, avec le spectre biologique brut global du type : $\mathrm{Th}>\mathrm{Hem}>\mathrm{GeO}>\mathrm{Ch}$. Les adventices therophytes se retrouvent dans les écotypes spécialisés, Elles s'adaptent dans des zones fortement perturbées et pendant les saisons non favorables, elles sont annuelles, et caractéristiques de régions sous une activité culturale avancée (Smith, 1912). En plus, les thérophytes prépondérantes dans un champ s'expliquent par une répartition de stock des semences des mauvaises herbes, qui se fait chaque année, cette situation est plus amplifiée par le mode de reproduction asexuée qui, résulte à la production de graines facilitant ainsi la dissémination. La dissémination se fait surtout par le vent et les insectes et en contact avec le sol directement elles germent. La stratégie de reproduction, comme chez les espèces des milieux cultivés est de type $" r »$.Ceci se traduit par un cycle court mais à forte capacité de reproduction. Ces adaptations sont typiques des plantes liées à des conditions pionnières : faible capacité de compétition, mais aptitude à produire en peu de temps un grand nombre de graines qui coloniseront d'autres sites perturbés. Les milieux instables temporaires, les premiers stades de friches des terrains abandonnés sont le domaine de prédilection de ces therophytes. Dans une parcelle cultivée, selon Jauzein en 1995, le rapport est en moyenne de $80 \%$ de therophytes pour $20 \%$ de geophytes. Les groupes de therophytes est le mieux adapté pour supporter les perturbations et c'est à leur stratégies de vie et de reproduction qu'elles doivent leurs succès. Elles ont une durée de vie courte, une croissance rapide, une maturité sexuelle précoce et produisent d'un grand nombre de semences. Ces espèces monocarpiques réalisent, en effet, un effort de reproduction très élevé. Les therophytes trouvés sont Achyranthes asper, Ageratum conizoides, Bidens pilosa, Galinsoga ciliata, Bothriocline longipes, Lactuca inermis et Oxalis corymbosa. Les geophytes trouvée sont Canna Indica, Cyperus distens, Myrabilis jalapa, Digitaria vestida. L'homogénéité spécifique de ces trois plantations s'explique par le fait qu'elles se trouvent toutes dans les conditions climatiques et édaphiques similaires étant donné que ces plantations ses retrouvent dans un même groupement qui est Bughore. La pluviométrie est un des facteurs responsable de la répartition des adventices dans un milieu, le type de sol est le deuxième facteur dans cette répartition. II n' y a pas une très grande distance du point de vu répartition pluviométrique et édaphique entre ces trois plantations (Agronomique, 2010). Le diagramme 
d'infestation montre que Bidens Pilosa et Galinsoga Ciliata sont des espèces majeures dans ces caféicultures. Elles sont aussi plus nuisibles (Tani et al, 2011), ceci s'explique par le fait que les conditions edapho-climatiques de la région sont favorable à ces mauvaises herbes de telle façon que ces plantes se retrouvent partout dans les trois plantations. Ces espèces apparaissent plus dans les milieux tellement anthropisés. Ce qui est prouvé par qui a montré que

\section{CONCLUSION}

L'inventaire floristique a été réalisé dans trois plantations du groupement de Bughore, il a été trouvé que Digitaria vestida, Galinsoga ciliata, Bidens pilosa, Botriocline longipes, Cyperus distens, Drymaria cordata, Setaria barbata et Ageratum conyzoides sont les espèces des asteraceae et poaceae qui sont plus

\section{RÉFÉRENCES BIBLIOGRAPHIQUES}

Balagizi, I.K., Ngendakumana S. ,Namegabe Mushayuma, M.H.,Trésor Mirindi Adhama, M. T., Bisusa,M.I., Baluku , B. et Isumbisho, M.2013. Perspectives de gouvernance environnementale durable dans la région de Lwiro (Sud Kivu, République Démocratique du Congo). Revue électronique en sciences de l'environnement disponible sur https://vertigo.revues.org/13826\#bibliography

Boudjedjou L., 2010. Étude de la flore adventice des cultures de la région de la région de Jijel, Mémoire de MAGISTER, Faculté des sciences, Département de Biologie, Université Ferhat Abbas-Setif, pp 155.

Delpech, R., 2006. Méthode pratique de la phytosociologie. Le réseau de la botanique francophone. pp. disponible sur http://www.telabotanica.org/page:methode_ph ytosociologique_sigmatiste?langue $=$ fr

Agronomique, N.D.R., 2010. Etude phytoécologique des adventices en cultures d'ananas (Ananas comosus ( $L$.) Merr .) dans les localités de Bonoua et $\mathrm{N}$ 'douci en Basse Côte d' Ivoire, pp.2367-2382.

Bénévoles, A.L.A.D.E.S., Notre café , une dose d'espoir pour la paix au Kivu Sopacdi.

Bourgeois, D.T. Le, La lutte contre les mauvaises herbes. , pp.649-670.

Dabou, D.E.S.I.A. et al., 2010. FLORE ADVENTICE SOUS PALMERAIE , DANS LA ZONE. , 22(1), pp.21-32. dans les caféicultures du Sud-Kivu, l'association avec d'autres cultures vivrière est une pratique couramment réalisée par les paysans. Les espèces sont souvent dangereuses à la culture principale. En ce qui concerne des espèces accidentelles du Site, peu nombreuses, elles ne présentent pas un problème pour la culture principale mais elles peuvent servir d'indicateurs écologiques.

caractéristiques des champs de caféier en pure. En plus, Ageratum conyzoidens, Achyranthes asper, Oxalis corymbosa, Crassophalum bumbese, Lactus spp. Cyperus distans, Commelina diffusa, Drymaria cordata. Sida acuta. Galinsoga ciliata et Bidens pilosa sont des espèces nuisibles dans ces caféicultures.

Flore, L.A. et al., 2004. COTONNIERES DE LA REGION DU WORODOUGOU ,.., 16(1981), pp.1-14.

Kouakou, J.N. et al., 2016. Importance relative des mauvaises herbes de la culture du maïs dans le département de $M$ ' Bahiakro [ Relative importance of weeds in maize cultivation in the M' Bahiakro department ]. , 17(3), pp.768778.

Lacs, G., 2014. La caféiculture et son incidence sur la transformation des conflits. , pp.1-72.

Mangara A., N'da Adopo A.A., Boraud M.K.N., Kobenan K., Lejoly J., Traore D., 2008. Inventaire de la flore adventice en culture d'ananas (Ananas comosus (I.) Merr.) dans la localité de Bonoua en basse Côte d'Ivoire. Agronomie Africaine 20 (1) : $23-35$.

Malakand, T., 2015. EXPLORATION AND INVENTORY ING OF WEEDS IN WHEAT CROP OF THE DISTRIC. , (October).

Onana, J. et al., 2007. ee des zones humides du NordCameroun: phytosociologie et utilisation pastorale To cite this version: La végétation herbacée des zones humides du NordCameroun Phytosociologie et utilisation pastorale.

Quee, D.D. et al., 2016. Journal of Experimental Biology and Agricultural Sciences WEED SPECIES DIVERSITY IN CASSAVA (Manihot esculenta Crantz ) MONOCULTURE IN ASHANTI REGION OF GHANA. , 4(2320). 
Reprenant, P., 1904. Etablir et comparer les spectres biologiques de plusieurs groupements végétaux. Sciences, F.D.E.S. et al., 2012. Les GROUPEMENTS des ADVENTICES des CULTURES dans la REGION d' ORAN.

Smith, B.Y.W.G., 1912. RAUNKIAER â€TM S " LIFEFORMS )" AND STATISTICAL METHODS.

Tehri, T. \& Garhwal, G.H.N.B., 2015. Weed flora of s.r.t. campus badshahi thaul tehri garhwal (h.n.b. garhwal central university..., (August).

Kazi T.C., Le Bourgeois,T. et , Francois Munoz, F., C., 2011. Contribution à l'étude des communautés d'adventices des cultures du secteur phytogeographique oranais (Nord-ouest algérien) : aspects botanique, agronomique et phyto-ecologique. in AFPP - VINGT ET UNIĖME CONFÉRENCE DU COLUMA JOURNÉES INTERNATIONALES SUR LA LUTTE CONTRE LES MAUVAISES HERBES DIJON - 8 ET 9 DÉCEMBRE 2010.HAL, archives ouvertes.13pages

Ronchi,C.P.,Silva, A A, Terra A A, Miranda, G V, 2015. Effect of 2, 4-dichlorophenoxyacetic acid applied as a herbicide on fruit shedding and coffee yield, in Weed Research, Volume 45, issue 1, pages 41-47.

Le Bourgeois et Marnotte, P..2002. La lutte contre les mauvaises herbes.663-684. In Anonyme.2002. Memento de l'agronome. CIRAD,GRET et Ministère des affaires étrangères.1698 page

Smith, W.G., 2013. Raunkiaer's "life forms)" and statistical methods. Inedit, Jstor, 11 pages
Pedel et Fabri,2012. État de l'art sur les indices existants concernant l'État Écologique des habitats benthiques du domaine profond. Convention MEDDTL - Ifremer pour la DCSMM - BEE, Convention 12/1219956/NYF.76 pages

Issouf,B., Adi Mama, D.F. R., NEUBA, K., J., K., Dossahoua T., Marjolein V., Brice S., Jean L. et Jan B.,2008. . Influence des actions anthropiques sur la dynamique spatiotemporelle de l'occupation du sol dans la province du Bas-Congo (R.D. Congo). Sciences \& Nature Vol. 5 N¹: $49-60$ (2008). 12 pages

Senterre,B. 2005, Recherches méthodologiques pour la typologie de la végétation et la phytogéographie des forêts denses d'Afrique tropicale. Inedit, Université Libre de Bruxelles (ULB), Faculté des Sciences, École Interfacultaire de Bioingénieurs, Laboratoire de Botanique systématique et de Phytosociologie, These de doctorat. 372 pages

Touré, A., J. Ipou Ipou, J., Adou Yao,C,.Y., Boraud,M,.K.N., et N'guessan, M.K.,2008. Diversité floristique et degré d'infestation par les mauvaises herbes des agroécosystèmes environnant la forêt classée de sanaimbo, dans le centre-est de la côte d'ivoire. Agronomie Africaine 20 (1) : 13 - 22 (2008).10pages 